\title{
“I'm Better Off on my Own”: Understanding How a Tutorial's Medium Affects Physical Skill Development
}

\author{
Shreyosi Endow \\ shreyosi.endow@mavs.uta.edu \\ The University of Texas at Arlington \\ Arlington, Texas, USA
}

\author{
Cesar Torres \\ cearto@uta.edu \\ The University of Texas at Arlington \\ Arlington, Texas, USA
}

\begin{abstract}
The shift towards distance learning brought forth by the pandemic has highlighted the shortcomings of teaching physical skills at a distance. With the emergence of new augmented and connected mediums, new opportunities arise for transferring physical skills that have resisted traditional documentation methods. However, there lacks a framework that allows tutorial authors to capitalize on a new medium's unique affordances rather than remediating existing tutorial conventions. Our work analyzes a body of tutorials rendered in various mediums for centering clay on a pottery wheel - a foundational skill that exemplifies the difficulties of physical skill transfer. Through the lens of McLuhan's "The Medium is the Message" we synthesize a taxonomy of medium conventions and themes derived from analyzing a body of centering tutorials and observation of how a tutorial's medium affects how learners develop physical skills. We leverage our findings to motivate design recommendations to inform how new mediums can support material practices.
\end{abstract}

\section{CCS CONCEPTS}

- Human-centered computing HCI theory, concepts and models; Empirical studies in interaction design; $H y$ pertext / hypermedia.

\section{KEYWORDS}

ceramics, tutorials, media analysis, skill transfer

\section{ACM Reference Format:}

Shreyosi Endow and Cesar Torres. 2021. "I'm Better Off on my Own": Understanding How a Tutorial's Medium Affects Physical Skill Development. In Designing Interactive Systems Conference 2021 (DIS '21), June 28-fuly 2, 2021, Virtual Event, USA. ACM, New York, NY, USA, 11 pages. https://doi.org/10.1145/3461778.3462066

\section{INTRODUCTION}

Shifting from in-person courses and training to distanced learning has magnified the need for better methods of transferring physical skills. Such skills are critical to studio [10,29], sports [20,26], and clinical $[4,35]$ practices, but are notoriously difficult to document

Permission to make digital or hard copies of all or part of this work for personal or classroom use is granted without fee provided that copies are not made or distributed for profit or commercial advantage and that copies bear this notice and the full citation on the first page. Copyrights for components of this work owned by others than ACM must be honored. Abstracting with credit is permitted. To copy otherwise, or republish, to post on servers or to redistribute to lists, requires prior specific permission and/or a fee. Request permissions from permissions@acm.org.

DIS '21, fune 28-fuly 2, 2021, Virtual Event, USA

(C) 2021 Association for Computing Machinery.

ACM ISBN 978-1-4503-8476-6/21/06 . .\$15.00

https://doi.org/10.1145/3461778.3462066 since many involve tacit actions that are performed intuitively and invisibly [21]. Due to the ambiguity of natural language, tacit skills are difficult to encode using verbal or written cues [25]. While video and other emerging mediums ${ }^{1}$ have increased the visibility of these actions, each medium can only offer a partial picture of the full experience [26].

Tutorials rendered in new mediums such as creative livestreams [6], $360^{\circ}$ videos, augmented reality trainings $[3,34]$, or wearables [1] are continuously evolving, offering new ways to relay tacit knowledge. However, understanding how to render content effectively given the affordances of a new medium is a moving target - a challenge that all new mediums faces and will continue to face [12]. There lacks a framework that can allow us to move towards new interactions for transferring tacit knowledge rather than continuing to emulate classic conventions like the synchronous step-by-step tutorial. Such a framework can help us develop instructional materials that offer new perspectives to bridging the tacit skill gap [12].

In this work, we take a step towards developing a unified mediumcentric framework for tacit skill transfer. Our work involves analyzing different instructional materials used for teaching the skill of centering clay on a pottery wheel - a process of adhering and positioning a mass of clay in the center of a spinning wheel that has been used to study tacit knowledge communication and skill transfer mechanisms in HCI $[1,15]$. As a foundational skill of thrown pottery [23], centering clay exemplifies the difficulties of skill transfer. Albeit a theoretically simple task, centering requires active and continuous attention and physical engagement of the hands, arms, torso, and feet. This situation limits a learner's input and feedback mechanisms - a traditional graphical user interface is inaccessible when hands are occupied, and even a voice user interface can introduce significant cognitive load. The amount of sensory information also oversaturates the information bandwidth of any one medium. While sensor-based methods and $360^{\circ}$ video can make perceivable information like the configuration, force, and pressure of the hands, they do not render the tacit information about where to attend to that traditional video offers; conversely, live video supports active discussion, yet is challenging to scale a live video platform to support individualized instruction. Every medium has developed conventions for presenting an instructional message that influences the experience of using and learning from tutorials.

Inspired by Marshall McLuhan's concept of "The Medium is the Message" [18], we perform a medium analysis of clay centering tutorials across book, video, and web forms. The analysis involves a

\footnotetext{
${ }^{1}$ We use the plural mediums to maintain focus on the connotation of a medium as a creative substrate, versus the plural media that is heavily associated with social media or news media.
} 
close reading of tutorial artifacts and a user study with five novices describing the experience of learning to center clay from these materials. From this analysis, we develop a taxonomy of medium conventions for representing information and a set of themes that describe continuing and alternative trajectories for augmented tutorials. We leverage our findings to motivate design recommendations to inform how new mediums can support material practices.

\section{RELATED WORK}

We describe related work within feedback modalities used in motor skill transfer mechanisms and augmented tutorials and motivate our task choice of centering clay through a review of established research on skill transfer in ceramics. We use the term "transfer" to refer to the exchange of knowledge and skills between individuals with different levels of expertise in performing a physical task.

\subsection{Skill Transfer within Ceramics}

The craft of throwing clay on a potter's wheel is rich in unspoken actions performed subconsciously or from muscle memory. Throwing clay has a steep learning curve and takes considerable practice for learners to fully grasp the intricacies of the task. Researchers are interested in communicating the tacit skills involved in the task and lowering the learning barriers for beginners. Liu et al. [15] visualized muscle activity through electromyography (EMG) data superimposed on video recordings of potters throwing clay on the wheel. The visualization was designed as a form of retrospective reflection and helped communicate the range of physical exertion required to manipulate the fast-moving clay. Bernal et al. [1] offered a real-time orientation and EMG intensity feedback through a light cue on a wristband by comparing the EMG and inertial measurement unit (IMU) readings of an expert's movements to a learner's movements. These feedback cues were found to assist learners in understanding individual steps more quickly and reduce the need to attend to video, yet required careful calibration to present useful corrective feedback. In our tutorial analysis, we distinguish these two approaches by the degree of creative sensemaking they elicit.

\subsection{Feedback Modalities in Motor Skill Transfer Mechanisms}

Researchers have used a wide range of feedback modalities to communicate motor skills. Force feedback modules are commonly used in handed tasks with a physical apparatus. Nakamura et al. demonstrated its effectiveness in improving the efficiency of a golf swing [20] while Yem et al. showed its value in guiding a medical student in operating a laryngoscope [35]. Vibrotactile feedback offers a lower power, mobile, and more wearable solution and ideal for activities with more sustained interactions such as sports and the creative arts. This vibrotactile strategy has been integrated on a violin bow to support musicians in violin practice and performance [10], on an armband to train novice badminton players in honing their long shots [22], and within the dance environment as a means of enabling motor skill transfer between dance experts and learners [2].

Although wearables can support remote interactions, integrating these devices within formal tutorials remains a challenge. These mechanisms are domain-specific, often embedded in the hand-held apparatus itself, requiring the design of bespoke devices that limits their generalizability outside their specific domain.

\subsection{Augmenting Tutorials}

Tutorial augmentation and authoring has a been a long-standing research area in HCI. Researchers are stepping away from linear tutorials in legacy mediums such as video and text and have created design methods that address how users learn to engage in new technological environments [28]. Ludwig et al. [17] used projection mapping as a visual user interface to support the collaborative appropriation of technologies such as 3D printing in non-expert contexts. Several works have looked at developing augmented reality tutorials from video tutorials to align user-tutorial viewpoints [34] and edit and combine multiple video tutorials to one novel AR tutorial [19]. Cao et al. [3] incorporated AR avatars to act as enhanced tutor presence for interactions that require large spatial navigation movements and full-body coordinative operations. Kumaravel et al. [30] developed a real-time bi-direction mixed reality telepresence system for improved interactions in remote instruction of physical tasks. However, AR hardware can be cumbersome, restricting users' field of view, sometimes occupying users' hands, and interfering with their focus and performance. Such restrictions limit AR's usefulness in attention-demanding tasks such as centering clay that occupy the hands and mind.

Researchers have designed tutorial authoring tools that improve a learner's engagement and agency in a physical task. MakeAware [27] went beyond linear, step-by-step tutorials by scaffolding situation awareness of a makerspace environment to guide a novice learner's perception, comprehension, and projection of a task. Ellustrate interactively generated material-specific tutorial instructions to match the unique design of a learner fabricating, testing, and debugging a conductive ink, thread, tape, or paint circuit [16]. To prevent a novice learner from following a step-by-step tutorial without understanding the process, Electrotutor [32] engaged the learner with interactive software and hardware verification tests to improve their comprehension. Other approaches have centered on developing communities of practice around tutorial creation for added social engagement [14]. Such communities of practice are essential in remote learning scenarios where a learner's access and engagement with his or her peers are limited.

Many tutorial authoring and editing tools strive to improve visibility and content presentation by updating tutorial visuals to maintain consistency [11], automatically generating static visual step-by-step tutorials from demonstration recordings [8], segmenting creative live streaming videos based on content [6] and removing repetition, silences, and mistakes from demonstration recordings [5]. However, in only making the successful and essential steps visible, tutorials often fail in providing a safe space for learners to grow from their failures. They do not capture the experience of the creative practice [13].

Gaps remain in understanding the role of tutorial's medium in a learner's experience. Our analysis of tutorial mediums identifies generalizable patterns that can inform augmented tutorial design directions. 


\section{METHODOLOGY}

Originating from McLuhan's concept of "The Medium is the Message" [18], medium in this work is interpreted as the material manifestation through which information is perceived [7]. This particular lens suggests when content or information is encoded into a medium, the medium has a significant influence on how the original information is interpreted, in some ways prioritizing the need to understand the medium over the content being rendered. This medium-centric lens is in sharp contrast to the focus of documenting and transferring domain knowledge in augmented tutorials and systems (e.g., violin playing [10], golf swings [20], and clay throwing [1]), which limits the generalizability of these works. In some cases, new tutorial mediums like popular cooking recipe websites emulate their closest medium counterpart (e.g., the physical cookbook). Often, these attempts of emulating a medium are incomplete and fail to make use of the new medium's unique affordances, which McLuhan refers to as medium props. Turning a page (a book medium prop) and following a hyperlink (a web medium prop) create different experiences in the learner's control of the flow of information within a tutorial. Instead, a medium-centric analysis identifies content-agnostic conventions that can generalize to new and emerging medium. Our approach mirrors McLuhan's Ryerson Experiment [18] where a message (a college anthropology lecture) was studied when rendered in multiple mediums (visual class notes, a radio lecture, as a television recording, and as a live lecture). The study observed the strong effect medium props had on student's performance on a comprehension quiz ${ }^{2}$.

Our analysis follows an equivalent pattern: we isolate a single message (the physical skill of centering clay on a pottery wheel) and analyze this message rendered as tutorials in three different legacy mediums (image/text, video, and web). Our focus on legacy mediums is aimed at making use of medium conventions, or shared design patterns that operate across different mediums, that have developed from decades of use and practice. Such conventions include the time-honored and pervasive step-by-step convention that prioritizes decomposing a message into cognitively processable chunks and can be rendered as an ordered list (text), a sequence of images (image), a video interspersed with step-based title slides (video), or a hyperlinked sequence of pages (web).

This analysis aims to identify the broader range of tutorialspecific medium conventions and organize them into a taxonomy usable by designers and engineers to motivate tutorial and tutorial system design decisions in augmented mediums (e.g., tutorials leveraging wearables, mixed reality, $360^{\circ}$ video, or live-streaming).

\subsection{Isolating the Message: Centering Clay}

In our analysis, the message, or content that is being communicated, refers to the process of centering clay on a pottery wheel. This message is rendered as an annotated step-by-step sequence in Figure 1. Centering clay on a potter's wheel involves manipulating clay so that it rotates symmetrically in the center of a spinning wheel; without this foundational step, uncentered clay leads to uneven or collapsing walls in thrown ceramics. The act of centering clay

\footnotetext{
${ }^{2}$ Such medium props included melody interludes in an audio lecture, preattentive cues in visual notes, or the ability to have an interactive $\mathrm{Q} / \mathrm{A}$ session with an instructor in a live lecture
}

engages multiple body parts and senses and has been a focus of research interested in skill transfer mechanisms and augmentation of craft practices $[1,15]$. Tutorials of centering clay are available in many different mediums, and the intricacies of the task itself exemplify the affordances and resistances of each medium.

\subsection{The Mediums: Artifact Analysis Set}

\begin{tabular}{|c|c|}
\hline Artifacts Analyzed & Analysis Focus \\
\hline \multicolumn{2}{|l|}{$\&$ IMAGE \& TEXT } \\
\hline $\begin{array}{l}\text { Filter: Explicit centering content; } \\
\text { physical copies } \\
\text { Keyword: "Beginner", "Clay Throwing" } \\
\text { Source: Library Archives, Online } \\
\text { Retailers } \\
\text { Total Content: ( } 8 \text { books; } 35 \text { pages) }\end{array}$ & $\begin{array}{l}\text { Image conventions } \\
\text { > Framing, Levels } \\
\text { Text conventions } \\
\text { > Treatments: Font, Size, Color } \\
\text { > Placement: Annotations, Captions } \\
\text { Layout conventions } \\
\text { > Bi-folds, montages, flow charts }\end{array}$ \\
\hline \multicolumn{2}{|l|}{$\triangleright$ VIDEO } \\
\hline $\begin{array}{l}\text { Filter: Most View Videos (10) } \\
\text { Keyword: "Centering clay } \\
\text { for beginners", "\#centering \#clay" } \\
\text { Source: YouTube, Instagram, } \\
\text { Total Video Duration: } 87.03 \text { min }\end{array}$ & $\begin{array}{l}\text { Video conventions } \\
\text { > Editing: Cuts/Transitions } \\
\text { > Shoots: Shot List/Framing }\end{array}$ \\
\hline \multicolumn{2}{|l|}{ SO WEB } \\
\hline $\begin{array}{l}\text { Filter: Web-unique conventions } \\
\text { Keyword: "Centering clay } \\
\text { for beginners" } \\
\text { Source: YouTube, Instructables, Blogs } \\
\text { Total Sources: } 15\end{array}$ & $\begin{array}{l}\text { Web conventions } \\
\text { > Hyperlinking } \\
\text { > Social reward systems } \\
\text { > Comments \& chats }\end{array}$ \\
\hline
\end{tabular}

Table 1: Table listing medium artifact set including the type of mediums, the number of artifacts analyzed and the focus of analysis

The selection of mediums to examine was based on emulating the experience of a novice learning thrown ceramics for the first time, focused on the resources that would be readily available to them (Table 1). We analyzed each artifact through the lens of three principal mediums: image and text, video, and the web. Table 1 describes the medium type, a breakdown of the artifacts analyzed, respective criteria for inclusion, and the specific elements of the artifact in focus.

The tutorials selected included eight physical books (35 pages), focusing explicitly on the chapters or pages describing the centering process. Ten centering clay videos were filtered based on their view count, and a total of 87.03 minutes of centering footage was analyzed. For web-based tutorials, we screened and studied 15 online tutorials, including the larger YouTube video platform, Instructables, and blogs. Due to limited variance in audio conventions in video-based tutorials (e.g., the instructors largely talked through steps and hand formations), we omitted audio-based props from our analysis.

\subsection{The Experience}

While a static analysis of the artifact set provided information on the props and conventions of the medium itself, it is important to also analyze how a tutorial's medium and content influence the perception and practice of a learner. Using three exemplar tutorial artifacts (Figure 2), we conducted a study observing how novice users perceive, make sense, and apply information from clay 


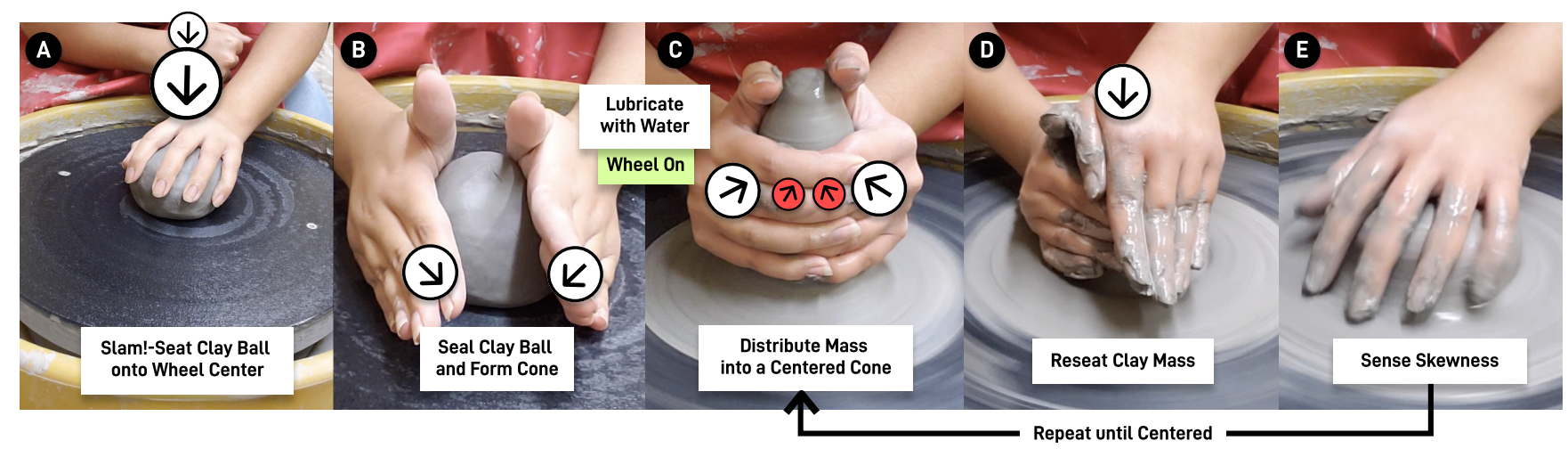

Figure 1: The Clay Centering Process (The Message): A) Slam (!) a wedged ball of clay onto the center of the pottery wheel; B) Seal the bottom edge of the clay mass to adhere the mass to the wheel; slap the mass into an initial cone shape. C) Squeeze the clay from the bottom to "cone up" using a hook-and-anchor hand position, being careful not to apply forces that would pull the mass off the wheel; D) Press the clay down with the heel of your palm to "cone down"; E) Hover fingers above the clay mass - if the fingers and wrist are steady, the clay is centered. Arrows annotate force directions; red-colored arrows indicate problematic forces; size indicates force magnitude.

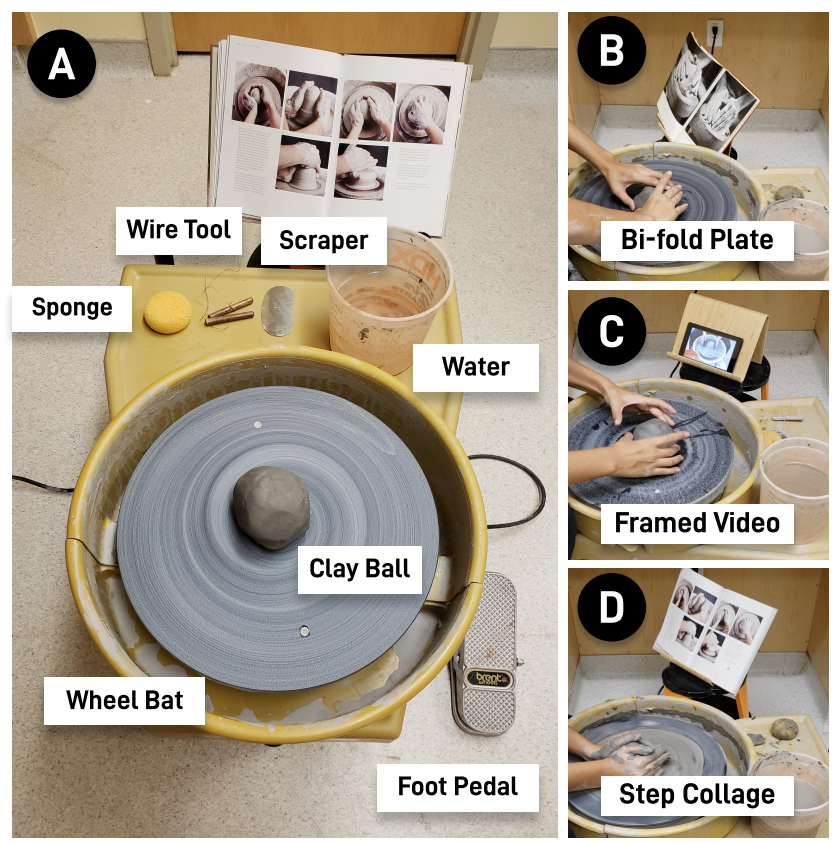

Figure 2: A. User study equipment and materials; (right) User study setup with three different tutorials - B. Wildenhain's Pottery, Form and Expression, C. Caswell's "How to center clay on the potters wheel", D. Carey's From Clay to Kiln: A beginner's guide to the potter's wheel

centering tutorials. This study provides context on the learners' experience and attitudes.

Participants. We recruited 5 participants (average age 24,3F, 2M) from a mailing list of Liberal Arts and Engineering students. We selected participants with no prior experience in throwing on a potter's wheel (although some participants had worked with clay before) to ensure that their experiences reflected those of a novice. We determined the eligibility and proficiency of participants through a pre-screening questionnaire. We also ensured that participants were comfortable being in a seated position and exercising their arms, back, and shoulders for the duration of the study. We invited participants individually to our studio space and limited each session to 2 hours, depending on how long the participants spent centering. Participants were compensated with a $\$ 10 / \mathrm{hr}$ Amazon Gift card.

Setup: Participants were given access to a Model C Brent Pottery Wheel controlled by a foot pedal. We provided participants with tools and materials required for centering including a bucket of water, a sponge, a wire tool, and a scraping tool (Figure 2). Participants had quick access to a clay sink and were provided an apron, eye protection, and hair ties to prevent injury and protect their clothing.

Protocol: A 5-minute warmup task was used to reduce novelty effects by familiarizing participants with the materials, hand tools, and operation of the pottery wheel. We provided a 450 gram wedged ball of clay. Participants threw the ball of clay on the center of the wheel bat and altered the wheel's speed to get a feel of the clay ball against their hands. The task was free play for 5 minutes. This task acted as a way to mitigate observer effects by establishing the study as more playful than experimental.

Participants were then asked to follow three tutorials on centering clay and were provided three pre-wedged 700 gram balls of moistened clay. The tutorials included a collage tutorial, a bi-fold plate tutorial, and a framed video tutorial. The task order was randomized and video recorded. Participants were asked to vocalize their thoughts out loud: specifically, the actions they were taking, whether their actions had their intended effects, and their overall feelings towards the process. The centered tasks were not assigned a time limit to reduce time stress, and a natural stopping point was 
chosen. After each tutorial, we asked users to rate their experience in a short questionnaire consisting of five-point semantically anchored Likert scale questions ( $1=$ Strongly Disagree, $5=$ Strongly Agree) probing tutorial effectiveness, expectations and outcomes, and satisfaction with their experience. After the session, we conducted a semi-structured interview with the participant to reflect on the process and gain additional insight into their overall experience. We designed questions to allow the participants to elaborate on some areas that addressed in the post-task questionnaires and others that we deduced were important from our artifact analysis including medium preferences, agency, intuition, and perceived differences from in-person instruction.

\subsection{Data Collection, Coding, and Themes}

When reading an artifact, we took notes in the form of quotes, images, screenshots, and video segments from the tutorials we analyzed. To seed our analysis, we first identified the medium props to identify the strengths and weaknesses of each tutorial medium in their representation of the centering process without getting distracted by the delivery of the content creator or the perception of the learner. We then identified medium conventions, or shared design patterns across mediums. The final collection of 33 medium props and 17 conventions were discussed among the paper authors and placed on post-its memos for collaborative coding and clustering.

From the user study, we reviewed think-aloud transcripts, questionnaire data, and interview notes, naming salient events and added them to our collection of post-its memos. We employed an axial coding strategy in our thematic analysis to establish connections between our static medium data, our experiential medium data, and an initial 7-element closed code set derived from craft literature: From The Craftsman [25], we used Sennett's categorization of instruction to characterize the tone, language and message of tutorials including sympathetic illustration, scene narrative, instructions through metaphors, and dead denotation. We describe Sennet's concepts in context with our resulting themes. From Centering in Poetry, Pottery and the Person [23], we incorporated domain-specific insights into the pottery and ceramics practice. Richards describes how a craft experience of pottery manifests in a potter's everyday life. We incorporate her three goals for teaching centering, including free perception, or letting the experience and form of the clay guide the potter's action, free initiative, or allowing time to play its course in building expertise without being obstructed by past negativities, and free participation, or the ability to come together as a community but also find individuality.

We used the codes derived from our artifact and experience analysis as well as the 7-element closed code set derived from craft literature to initially cluster and organize medium conventions and props, before iteratively developing new codes from relationships between medium conventions and experiences. We then identified themes from these patterns and relationships and synthesized them into design principles to guide the development of new medium tutorials.

\section{TAXONOMY OF MEDIUM CONVENTIONS}

In order to organize and communicate our medium analysis findings, we used our conceptual medium model to motivate a medium convention design space. In particular, each artifact was viewed through the medium-specific lens of image and text, video, or web; medium conventions, or common patterns, were arranged in a Venn-diagram (Figure 4). For instance, when reading a YouTube

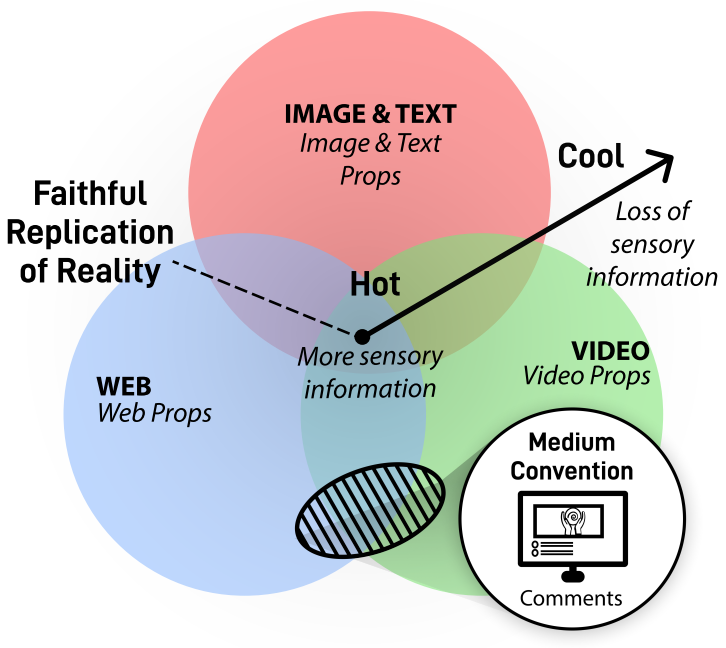

Figure 3: A conceptual model of McLuhan medium terminology used to code medium conventions. Each tutorial artifact is viewed through the lens of a specific medium. When considering a YouTube video tutorial, we plot the medium convention of the comment at the intersection of video and web since these web-specific comments contain timestamps that can control video position.

tutorial through a video medium lens, we noted video-specific properties (video props) such as the ability to start and stop the content. When viewing that same artifact through a web medium lens, we noted web-specific viewer-provided comments. These comments in particular had a linked timestamp that could control the video's position - we thus placed comments at the cross-section of video and web mediums. We excluded comments from the image and text cross-section since the actual text in the comments did not engage with image \& text -specific props (e.g., text or image treatments). We used the degree of cross-section as an initial assessment of McLuhan's hot and cool medium distinction - the more sensory information is provided, the less amount of sensemaking the user must engage with. As a grounding example, YouTube comments do not provide information to the user such as tone or the identity of the comment's author; this requires the tutorial user to actively interpret these qualities to make sense of the comment's value, veracity, or relevance.

From the Venn-diagram, we derived a taxonomy intended to be used by designers to navigate different design decisions when developing tutorials in new mediums. Medium conventions were plotted along two dimensions: we plotted each convention according to the level of creative sensemaking they elicit (displayed on the yaxis). This placement was also motivated from observing how users 


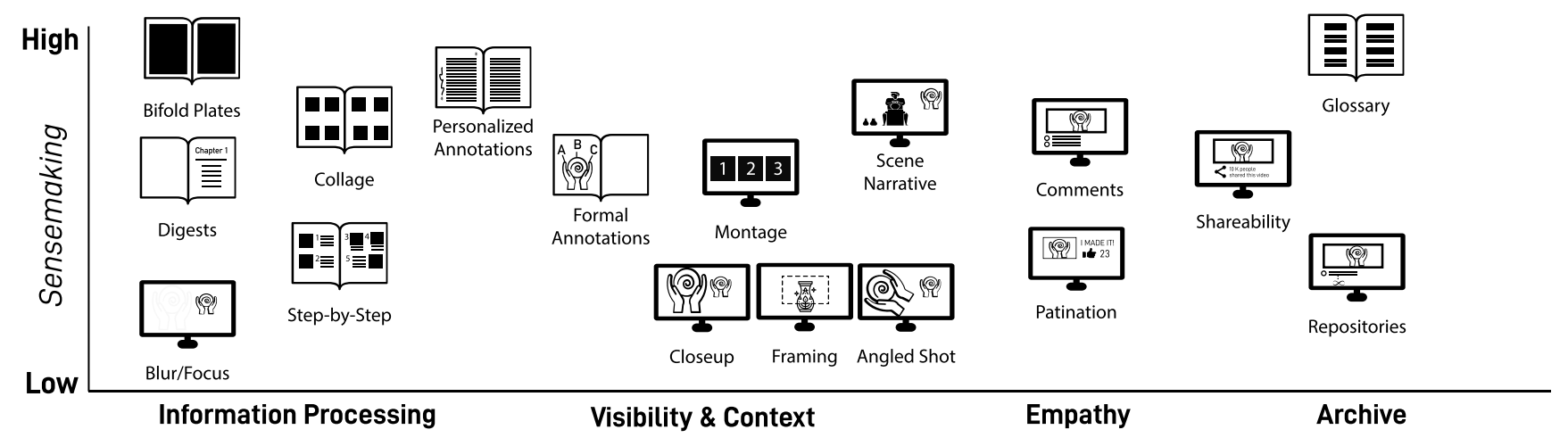

Figure 4: Medium Conventions Taxonomy. Conventions are plotted along their respective roles in creative sensemaking ( $x$ axis) and their degree of creative sensemaking (y-axis).

in our user studies experienced a subset of the tutorials analyzed. Conventions with sparse details that engage only a single sensory modality were ranked with high creative sensemaking(e.g., bifold plates); in contrast those that provided users with a "complete" picture were ranked as requiring low creative sensemaking (e.g., blur and focus shots in video). We then used associations, generated when coding these medium conventions, to organize medium conventions thematically by their major role in the tutorial experience (displayed on the x-axis) and described below.

Information Processing. Several medium conventions have a primary goal of rendering information to be easier to cognitively process. For example, the blur/focus conventions removes distractors by reducing the saliency of peripheral stimuli. In image and text, this corresponds to image treatements like reducing the saturation of a subset of the environment to drive the eye towards a region-of-interest. In video, we see blur/focus more naturally accessed through motion cues that, for instance, draw the eye towards a changing form or gesturing hand. Other notable medium conventions in this space rendered hierarchies of information, from digests in the form of chapters or overviews, to breaking down complex process into linear step-by-step instructions. While these conventions make it easier to process information, the amount of information they encode is unique. For instance, bifold plates, or two images juxtaposed next to each other reduces cognitive processing needs - afterall, two images are easier to process and remember than an entire step-by-step tutorial; however, the overall information explicitly communicated is limited. Instead, a tutorial user must engage in significant sensemaking activities to analyze, compare, and contrast the two images to discern the implicit instructions (e.g., hand and body positions, before-and-after states) that would normally be rendered in text or spoken instruction.

Visibility and Context. The aim of visibility and context conventions are to bring higher fidelity to a tutorial user's situation to allow easier access to the information as it is needed. For instance, closeup,framing, and angled shot emulate the viewpoint of the user or direct the user to attend to a particular area so as to make sure the information that is being relayed is relevant and useful. While more common in video-based mediums, we see variants in personalized annotations, such as highly voted comments annotating portions of a video to focus on or the penciled writings in a book providing more practical information alongside the content it pertains to. While information processing conventions try to remove information that is thought to be unnecessary or distracting, contextual conventions show information outside the scope of the traditional tutorial (scene narrative). These details often contain tacit information (e.g., where to buy clay, how much water to use, the force to apply, and failed attempts) that a user must actively parse to answer the unique problems or situations they face.

Empathy. Empathy conventions largely aim to promote an experience of shared struggle or effort, especially as a means of allowing the tutorial to resonate with users' skill level. While comments, or conversations shared amongst viewers of a tutorial, are the most common way to establish a common frame, some mediums couple comments with the evidence of struggle or engagement as a form of patination. Patination is more salient in web-based mediums, for example in the promotion (e.g., like, heart, upvote) of comments attached to videos or questions within community forums that make visible the difficulties that users face.

Archive. Archival medium conventions support the sharing, dissemination, and access to tutorials or elements of tutorials. Formal archives, or repositories, provide more complete curation of techniques and skill development by organizing community submissions (e.g., YouTube video ranking). Shareability refers to explicit sharing mechanisms, notably at different granularities of information (e.g. share full tutorial link versus share timestamp or subsection link). Glossaries serve as archives of concepts, materials, and techniques, but require users to connect these elements to tasks or context of use.

\section{THEMES}

We present five themes that emerged from the empirical data collected from the user study juxtaposed against the medium conventions taxonomy. We first identify concrete occurrences of medium conventions and then describe relevant experiences encountered by users in our study. 


\subsection{T1: Ambiguity invites self-defined success metrics}

In our analysis, several conventions had the common role of removing information, which may seem counterintuitive to the goal of transferring a physical skill like centering. In contrast to conventions like digests that "compress" the message to satisfice the information bandwidth of human cognition, we instead found conventions that introduce ambiguity by selectively remove entire elements of the message. As an example, one tutorial rendered the clay centering message on two black-and-white bifold plates, or large juxtaposed images on opposite sides of a page fold [33]. The images were captioned with two simple instructions describing the hand-position required to cone up. While providing little explicit instruction, the large visually examinable images invited users to make their own connections between the two depictions, parsing the change in position of hands, the shift of the form, and any other minutiae in the surroundings. The images and short captions are easier to commit to memory, providing consistent feedback to the user through recall. This high creative-sensemaking tutorial conveyed just enough information to get a novice started but entrusted them to derive their own success metrics through trial-and-error instead of setting predefined goals.

Experience. In our study, we observed that ambiguity encouraged some learners to use their intuition which, in turn, motivated them to continue with the experience. Two out of five users raised questions about the ambiguity of the written language and the static, single-angle images in the collage and bi-fold plate tutorials:

U4 The book says to add a good amount of water. I don't know what they mean by a good amount.

U3 The image doesn't really show how much force she is applying. I'm not sure at what point it will break.

Rather than being intimidated by the ambiguity, the users approached the process in a trial-and-error fashion by adding water and applying force until it "felt right." Due to the randomized ordering, only one user, U2, started with the bifold plates, which provided the least amount of information about the actual centering. We observed that although the user began by trying to mimic the hand position in the plates, they soon derailed from the tutorial by opening the uncentered clay up into a cylinder shape. Within six minutes, the user informed us that they thought the clay was centered and wanted to move on to the following tutorial. This lack of critical interpretation was a trend in the text tutorials where users felt confident that they had successfully centered the ball of clay despite an incorrect centering attempt, highlighting the variability in users' definition of success when following a high-creative sense-making tutorial.

In one instance, self-defined success metrics led to doubt after several "failed" attempts, hindering the enjoyment of the process.

U5 I remember the video saying, "Here's a good rule to follow: if you're not sure, then it's not centered." Thinking back, [my attempts] was probably centered, but I was unsure and kept redoing the steps. I got frustrated.

\subsection{T2: Scene narratives resonate with a personable narrator}

Although tutorials appear impersonal compared to in-person instruction, we saw different relationships between learner and author in tutorial artifacts. For example, Jonthepotter ${ }^{3}$, a popular pottery channel on YouTube, takes the viewer around his studio as he shows how to perform many different types of ceramic tasks. The relationship becomes more informal, providing elements of a scene narrative that reveals information that goes beyond the original message but also provides context and backstory. The view of Jonthepotter's time-worn pottery wheel, speckled with clay slip or the organized chaos in the background studio, provides opportunities for a learner to relate to the messier realities of working with clay. The scene narrative influences the credibility of the narrator and breaks the formal boundary of the traditional master-apprentice model. We saw scene narratives manifest in different ways, for instance, as snapshots organized as collages in Instagram profiles or montages in the beginning segment of a video. The qualities also surface more saliently through patination mechanisms like views, likes, and subscribers, or more explicitly Instructables's "I Made It" interaction ${ }^{4}$. Unlike many formally published tutorials, scene narrative shows context, often portraying errors and failures that make them personable, matching and empathizing with the difficulties that most novice users encounter.

Experience. Since we used preselected tutorials, a user's personal narrative preference was not reflected in the user study. The plate tutorial relied heavily on image to convey the tone of the process; the black-and-white photographs communicated a romantic return to the artisan, yet the caption was precise and didactic. While the plate tutorial was appreciated as "neat" (U1) and "exploratory" (U2) by some users, others placed mistrust in the author:

U5 The hand position described was unlike the other tutorials.

I feel less inclined to follow it. I'm not sure whether it would yield my desired goal.

The framed video tutorial was similarly cropped to the potter's hand, didactic in tone, with only a voice betraying its more personable qualities. While the video tutorial was found to be "beginnerfriendly" (U4), it was perceived as requiring more background knowledge:

U3 I would need some knowledge of the process for this to work

\subsection{T3: Material encounters improve agency}

While hand gestures, forces, speed, and the overall feel of the centering process were common information highlighted across all medium types, we encountered instances where learners were encouraged to center with their eyes closed and eliminate all distractions to amplify the sense of touch. We found this concept rendered in mediums such as videos that removed didactic narration and instead used blur and focus and close up to center the viewer's eyes on skin-and-clay interactions. However, outside the scope of this analysis, such videos often employed high-quality audio that amplified the unique sonic signature of each centering task. For example, in her "Centering clay for beginners" video, Lea Townsend

\footnotetext{
${ }^{3}$ https://www.youtube.com/jonthepotter

${ }^{4}$ https://www.instructables.com/Thowing-a-Pot-101/
} 
removed any background noise and angled her camera such that only the potter's wheel and her hands were visible on the frame. She advised her viewers to eliminate as many distractions as possible while centering, encouraging them to let the clay guide their actions.

Experience. While not rendered in the tutorials users experienced, we encountered several instances where users listened to their haptic perception to motivate their actions.

U1 My hands are getting hot, so I will try to add more water.

U4 The clay is wobbling like a broken washing machine, so clearly it is not centered!

U2 My hands are no longer dragging against the wheel, I must be getting it right!

Users notably referenced tutorials only after periods of difficulty; the collage and framed video tutorials both suffered from synchronization issues. All five users aimed to follow along with the steps of the tutorial author, stopping the video when it went outof-sync or when they began struggling with the clay. Although most users rewinded to a previous segment after attempting to fix their mistakes, U1 kept the tutorial turned off as they continued centering. Upon asking to reflect on their decision, U1 said:

U1 I'm better off on my own. I wanted to focus, and I felt like the tutorial was throwing a lot of information at me that wasn't useful at the time.

His actions focused instead on probing different gestures, hand positions, and forces upon the clay to build familiarity of how it reacts and re-interpreting and contextualizing the instructions of the previous step.

\subsection{T4: Tutorials skew perception of effort}

Images and videos of centering clay depict the process as low effort. The framing and selective curation of shots in the collage, promote a false fluidity of the process. To keep tutorials short and attractive, images and videos only demonstrate the successful attempts, creating an illusion of ease about the process. Some videos allot segments to discuss mistakes and their respective solutions, while texts generally sprinkle in warnings about actions that can lead to undesirable results within the steps. For example, in the book From Clay to Kiln by Stuart Carey, the centering steps are accompanied by photographs of the potter's hands performing the steps. Although there are some warnings in the written text, the static, aesthetically pleasing images depict the steps once they have been accomplished, portraying the success rather than the effort to perform the step or any number of things that could go wrong while performing the step.

Experience. In early attempts, users were eager to mimic the images of the books and the actions demonstrated in the video. Users expressed confusion when their efforts led to different results than in the tutorials. Some users interpreted the disconnect as a mistake, modifying their actions which exacerbated the gap in their results.

U1 The clay isn't coning up as easily as it does in the video. It's coming off in my hands, and the ball is getting smaller. Maybe if $\mathrm{I}$ try adding more water...
Users were wary of the way the collage and framed video tutorials anticipated mistakes. The video tutorial demonstrated common errors and solutions towards the end of the video. Most users never reached the segment, being too caught up in the centering portion. Upon realizing that, the users were disappointed.

The collage tutorial interleaved the mistakes and solutions of centering with the steps. Users rarely shifted their focus to reread the text during centering, citing a reluctance to search for the information.

U4 I wish the images showed the mistakes instead of these perfect workflow photos. I have to go reread the text to find my mistakes, and even then, I'm not sure if what they are describing is what's happening to my clay.

These insights suggest that the skewed perception affects a user's progress, causing doubt that delays their success.

\subsection{T5: Hands-on tasks limit interactions with tutorial technologies}

Centering clay is an inherently messy task that limits interactions with the tutorial device (e.g., a printed page, book, phone, or laptop). Interactions while centering with books where the entire process is not confined to a centerfold risked water or clay damaging the pages. For example, John Colbeck's The Technique of Throwing covers centering over multiple pages, often referring to images and text from a previous page requiring a reader to turn to that page to grasp the steps thoroughly. Furthermore, touch screens of devices displaying a video or web-based tutorial may not recognize the touch gesture due to the clay coating the fingers, limiting users from accessing medium props such as zoom, fast-forward, or pausing the video.

Experience. Users were hesitant to interact with the tablet and books during centering. Only two users wanted to turn the page of the collage tutorial during centering and asked for our help. On the other hand, all users needed our assistance in rewinding, pausing, or fast-forwarding the video.

U1 I felt like the lady in the video was talking very fast, so I

had to pause and rewind it initially.

In contrast to U1, some users found the pace of the video tutorial perfectly suited for them but struggled with the books due to not being able to turn the pages. The difference in opinions arose from a difference in learning style as well as pace and content preference.

\section{RECOMMENDATIONS FOR DESIGN}

Using the taxonomy. New mediums such as augmented reality or the Internet of Things present unique affordances that are difficult to translate from legacy mediums like text, image, and video. We designed our taxonomy to identify patterns across mediums that could be used to inform design directions that prioritize creative sensemaking. The aim is not to apply a trusted convention, such as the step-by-step, to a new medium because it has always worked, but to look at how the convention can be used to anticipate a learner's experience. For example, if a new medium arises that can display all the motions of the hand as a person is centering, it would be beneficial to look at some higher sensemaking conventions (top region of the taxonomy) and consider how the tutorial 
could give the learner more opportunities to make sense of the hand's motions. The taxonomy identifies a few design trajectories - this motion-enhanced centering tutorial could present the space of hand motions through a glossary, pose the user's motion and the author's motion side-by-side (bifold plates), or selectively annotate parts of a video with personalized annotations marking the different hand motions used as opposed to using exhaustive formal annotations that mark every hand motion.

We leverage the themes and taxonomy of conventions identified from our medium analysis to discuss how they can inform tutorial design directions for learnability, glanceability, reuse, and improving learner efficacy.

\subsection{Communicating safety boundaries}

Create an environment that conveys the potential actions of a task and their boundaries of operation to encourage an open-ended construction of knowledge.

Creative practices and constructionist learning theory agree that activities that encourage open-ended interpretation help learners to more actively construct knowledge [9]. Richards [23] explains this as a deliberate learning strategy, limiting the amount of handholding and instead centering the learning process on trial and error. One critical consequence of introducing ambiguity (T1) is that by placing the burden on the user to rely on their own senses and judgment, they, in turn, develop their own perceptions of success and failure [23]. However, it is not always possible to control access to tutorials with this pedagogical aim - as we saw in our user study, the ordering effects influenced how the bi-fold plate tutorial was perceived. Users who started with the plate tutorial found the experience exploratory and enjoyable. However, users who started with tutorials that provided more explicit information found the plate information unsatisfactory, falling back to referencing the steps and milestones from previous tutorials.

Many media conventions move away from ambiguity, instead aiming to improve visibility and make consuming the information more effortless. These include strategies for improving the hierarchy of saliency through digests, blur and focus, or through cognitive chunking like the step-by-step and the collage. It is important to distinguish that introducing ambiguity into a tutorial is not about causing frustration from the lack of information but instead communicating what constitutes a safe environment and the set of available actions that one can explore. As a motivating example, new wearable sensor-based technologies could allow tutorial authors to record their tacit actions (e.g., application of pressure by their hands), which would allow a tutorial user to obtain immediate feedback as to "correct" or "incorrect" hand positions. Instead, such a tutorial could leverage this action as a site for constructionist learning by only communicating the bounds of pressure to apply Rather than requiring active attention from the user, this interaction allows for a larger reliance on haptic perception between the user and clay that helps build a mental model of how clay reacts to pressure. This more direct engagement with clay and decoupling of the tutorial medium creates an environment for material conversations (T3).

\subsection{Dissolving perceptions of the genius}

Communicate effort to remove illusions of perfection and set realistic expectations.

Scene narratives serve to prepare a learner's mindset for the task at hand and are often achieved through personal anecdotes about the task experience [25]. Narratives benefit from an engaging and credible narrator, or they fail to garner a learner's attention, trust, and commitment. In book-based tutorials, the responsibility of establishing narrative credibility is mostly dependent on the author's writing tone or the larger perception of the rigor and prestige of book publishing. While images can aid in painting a scene, most books portray centering in static, professional setups. A singular perspective of an author's hands guiding a clay ball on a clean pottery wheel conveys little about the author compared to, for example, a $360^{\circ}$ view of the author's personal studio setup.

Although we encountered tutorial authors attempting to resonate with a novice's experience (Sennet's sympathetic illustration [25]), we found that tutorials rarely demonstrated mistakes. We encountered that humanizing the narrator and dissolving the impression of the artistic genius invited greater agency and attention on the part of the learner. Researchers have looked at the merits of visualizing failure as a motivator for a novice learner's growth in the learning process $[13,31]$. Demonstrating failure, however, is information that is only needed opportunistically and rarely can be anticipated by a tutorial author. Presenting failure information upfront carries the same quality as an instructional manual, where a learner must navigate to the information that is needed in the moment. As a grounding example, patination of user activity, such as encoding the view history of a video, could indirectly provide an indication to new viewers where difficulties occur; for tutorial authors, this activity can be used to identify disconnects between the author's experience and the learner's and inform personalized annotations that direct learners to supplemental materials that provide additional context. These failure resources, when gathered together, can form empathetic archives including glossaries to more formally curated repositories of failure.

\subsection{Redirecting feedback to perception}

Foreground a user's ability to perceive and make sense of their activity over interactions with the tutorial medium.

Centering is a body-centric task that engages visual, auditory, and haptic perception. While users had explicit tutorials, a different type of instructional activity occurred between the user, the clay, and the wheel. As introduced by [24], this reflective conversation with a material serves as a basis for how practitioners learn and guide creative practice. The clay in this situation communicates its conditions to the learner through different cues - the friction between a drying clay causes the learner to apply uneven force. Richards [23] refers to this metaphoric language spoken between the hands and the clay as "skin ears." One of her goals in teaching centering was to foreground skin-material interactions to be the main feedback mechanism as a learner develops their centering skills.

When we consider tutorials rendered in web or video mediums or extrapolate to augmented reality mediums, the interactivity of these mediums is at odds with material-based interactions. In our 
user study, a tutorial author's voice issuing comments to address ambiguities or resonate with the learner served largely as a distraction. The need to attend to the tutorial across mediums (e.g., turn a page, pause a video, or jump to a playlist) disrupted workflows, leading to one user's comment and our work's title "I'm better off on my own". Tutorials can benefit from foregrounding these material-based interactions and the body's natural abilities to perceive this rich feedback. For instance, tutorials could leverage blur and focus to reduce peripheral noise and instead amplify clay-skin interactions: in an audio-based medium, through a microphone amplifier; in a video-based medium through slow-motion, focused shots. Consistent repetition can allow the learner to naturally develop their "ear" as a form of creative sensemaking that is useful beyond the scope of a particular task within a practice.

\subsection{Maximize spatio-temporal control}

Prioritize user control and sensemaking abilities gained from a tutorial's flow of information.

A common challenge across tutorial mediums is the need to synchronize the message to the unique situation at hand. Information processing conventions approach this challenge by maximizing glanceability, or the ability to localize and transfer relevant information in the timespan of a glance. Researchers have developed tools to improve the glanceability of tutorials. For example, DemoCut [5] condensed repetition and silent segments in video tutorials to create digests which would help a user get the higher-level picture of a task and promote agency. While the trend in these systems is to provide better message compression, visibility, and viewpoint control [3, 19, 34], they also limit a user's spatio-temporal control. More passive mediums like book or web tutorials maximize a learner's spatio-temporal control - the tutorial will only advance when a learner turns a page or when they scroll or advance to the next step. However, these forms of spatio-temporal control all depend on physical input with the tutorial medium, which introduces context-switching demands on the learner to navigate the tutorial space alongside attending to the physical task at hand(T5) Centering clay exacerbated this issue, especially since muddy hands rarely mix with either passive or active technologies.

One natural solution to the synchronization challenge is leveraging voice-user interfaces or, to a greater extent, brain-computer interfaces to expose non-hand based input mechanisms and improve follow-along capabilities. However, reducing the need to control a tutorial's flow of information poses a more usable and resilient strategy. We encountered passive text and image-based tutorials as having the most mature mechanisms to address synchronization through a collage of images depicting the centering process on a single sheet, tutorial authors spatialized temporal control allowing a learner's gaze to access relevant information, look ahead and behind, and not require physical input. Video mediums, especially those used in distanced learning, could enhance the collage, providing repetitive looping mechanisms to allow the learner to form and resolve hypotheses of how physical actions were planned and resolved.

\section{LIMITATIONS}

Separating the tutorials to purely text, photographs, video, audio, or web was limited since each tutorial depends on multiple mediums to transfer information. We instead extracted the unique properties of each medium type through medium props and observing how they lead to more generalizable medium conventions. Our user study was not able to fully emulate the experience of following a webbased tutorial. Web-based conventions such as comments, likes, or sharing with a larger community, or conversing with the tutorial author. To assess the experience of web-based conventions, we purposely introduced a YouTube video tutorial with a conventionrich comment section and explicitly asked the participant to review this content.

\section{CONCLUSION}

In this work, we used McLuhan's "The Medium is the Message" as a lens to analyze how the medium in which a tutorial is rendered affects how users develop physical skills. Focusing on the physical skill of centering clay in thrown ceramics, we analyzed 33 tutorials rendered across the book, video, and web mediums. We generated a taxonomy of medium conventions to describe common patterns of relaying information. In a user study with five participants, we observed how these medium conventions affect the experience and perception of learning. We synthesized themes from our analysis that describe how creative sensemaking is more salient in mediums that provide the user with less information and provide opportunities for users to resonate with the tutorial author. We pose a set of design recommendations to motivate design trajectories of emerging new mediums and augmented tutorials that can engender new participation and engagement with physical practices.

\section{ACKNOWLEDGMENTS}

We thank the anonymous reviewers for their insightful feedback, the users who participated in our study, and the peer support from The Hybrid Atelier at UTA.

\section{REFERENCES}

[1] Guillermo Bernal, Dishaan Ahuja, and Federico Casalegno. 2015. EMG-based biofeedback tool for augmenting manual fabrication and improved exchange of empirical knowledge. In Proceedings of the XVI International Conference on Human Computer Interaction. 1-8. https://doi.org/10.1145/2829875.2829932

[2] Hector M Camarillo-Abad, J Alfredo Sánchez, and Oleg Starostenko. 2020. An environment for motor skill transfer based on wearable haptic communication. Personal and Ubiquitous Computing (2020), 1-25. https://doi.org/10.1007/s00779020-01425-z

[3] Yuanzhi Cao, Xun Qian, Tianyi Wang, Rachel Lee, Ke Huo, and Karthik Ramani. 2020. An Exploratory Study of Augmented Reality Presence for Tutoring Machine Tasks. In Proceedings of the 2020 CHI Conference on Human Factors in Computing Systems. 1-13. https://doi.org/10.1145/3313831.3376688

[4] Amine Chellali, Cédric Dumas, and Isabelle Milleville-Pennel. 2010. WYFIWIF: A haptic communication paradigm for collaborative motor skills learning.

[5] Pei-Yu Chi, Joyce Liu, Jason Linder, Mira Dontcheva, Wilmot Li, and Bjoern Hartmann. 2013. Democut: generating concise instructional videos for physical demonstrations. In Proceedings of the 26th annual ACM symposium on User interface software and technology. 141-150. https://doi.org/10.1145/2501988.2502052

[6] C. Ailie Fraser, Joy O. Kim, Alison Thornsberry, Scott Klemmer, and Mira Dontcheva. 2019. Sharing the Studio: How Creative Livestreaming Can Inspire, Educate, and Engage. In Proceedings of the 2019 on Creativity and Cognition (San Diego, CA, USA) (C\&C '19). Association for Computing Machinery, New York, NY, USA, 144-155. https://doi.org/10.1145/3325480.3325485

[7] Verena Fuchsberger, Martin Murer, and Manfred Tscheligi. 2013. Materials, materiality, and media. In Proceedings of the SIGCHI conference on human factors in computing systems. 2853-2862. https://doi.org/10.1145/2470654.2481395 
[8] Floraine Grabler, Maneesh Agrawala, Wilmot Li, Mira Dontcheva, and Takeo Igarashi. 2009. Generating photo manipulation tutorials by demonstration. In ACM SIGGRAPH 2009 papers. 1-9. https://doi.org/10.1145/1576246.1531372

[9] James G Greeno, Allan M Collins, Lauren B Resnick, et al. 1996. Cognition and learning. Handbook of educational psychology 77 (1996), 15-46.

[10] Tobias Grosshauser and Thomas Hermann. 2009. Augmented haptics-an interactive feedback system for musicians. In International Conference on Haptic and Audio Interaction Design. Springer, 100-108. https://doi.org/10.1007/978-3-64204076-4_11

[11] Andrew Head, Jason Jiang, James Smith, Marti A Hearst, and Björn Hartmann 2020. Composing Flexibly-Organized Step-by-Step Tutorials from Linked Source Code, Snippets, and Outputs. In Proceedings of the 2020 CHI Conference on Human Factors in Computing Systems. 1-12. https://doi.org/10.1145/3313831.3376798

[12] Jim Hollan and Scott Stornetta. 1992. Beyond being there. In Proceedings of the SIGCHI conference on Human factors in computing systems. 119-125. https: //doi.org/10.1145/142750.142769

[13] Joy Kim, Avi Bagla, and Michael S Bernstein. 2015. Designing creativity support tools for failure. In Proceedings of the 2015 ACM SIGCHI Conference on Creativity and Cognition. 157-160. https://doi.org/10.1145/2757226.2764542

[14] Benjamin Lafreniere, Tovi Grossman, and George Fitzmaurice. 2013. Community enhanced tutorials: improving tutorials with multiple demonstrations. In Proceedings of the SIGCHI Conference on Human Factors in Computing Systems. 1779-1788. https://doi.org/10.1145/2470654.2466235

[15] Yilin Elaine Liu, Michael Nitsche, and Benjamin N Sugar. 2019. Reflection on Tacit Knowledge-Effect of Providing EMG Visualization on Reflections on Throwing Clay. In Proceedings of the 2019 on Creativity and Cognition. 619-625. https: //doi.org/10.1145/3325480.3326576

[16] Joanne Lo, Cesar Torres, Isabel Yang, Jasper O'Leary, Danny Kaufman, Wilmot Li, Mira Dontcheva, and Eric Paulos. 2016. Aesthetic electronics: Designing, sketching, and fabricating circuits through digital exploration. In Proceedings of the 29th Annual Symposium on User Interface Software and Technology. 665-676. https://doi.org/10.1145/2984511.2984579

[17] Thomas Ludwig, Michael Döll, and Christoph Kotthaus. 2019. " The Printer is Telling Me about Itself" Supporting the Appropriation of Hardware by Using Projection Mapping. In Proceedings of the 2019 on Designing Interactive Systems Conference. 331-344. https://doi.org/10.1145/3322276.3322342

[18] Marshall McLuhan and Quentin Fiore. 1967. The medium is the message. New York 123 (1967), 126-128.

[19] Peter Mohr, David Mandl, Markus Tatzgern, Eduardo Veas, Dieter Schmalstieg, and Denis Kalkofen. 2017. Retargeting video tutorials showing tools with surface contact to augmented reality. In Proceedings of the $2017 \mathrm{CHI}$ Conference on $\mathrm{Hu}$ man Factors in Computing Systems. 6547-6558. https://doi.org/10.1145/3025453. 3025688

[20] Takuto Nakamura and Hideki Koike. 2020. Golf Club-Type Device with Force Feedback for Modifying Club Posture. In Extended Abstracts of the $2020 \mathrm{CHI}$ Conference on Human Factors in Computing Systems. 1-7. https://doi.org/10.1145/ 3334480.3383024

[21] Claudia Núñez Pacheco and Lian Loke. 2017. Tacit narratives: Surfacing aesthetic meaning by using wearable props and focusing. In Proceedings of the Eleventh International Conference on Tangible, Embedded, and Embodied Interaction. 233242. https://doi.org/10.1145/3024969.3024979

[22] Ashutosh Raina, TG Lakshmi, and Sahana Murthy. 2017. CoMBaT: Wearable technology based training system for novice badminton players. In 2017 IEEE 17th International Conference on Advanced Learning Technologies (ICALT). IEEE, 153-157. https://doi.org/10.1109/ICALT.2017.96

[23] Mary Caroline Richards. 1989. Centering in pottery, poetry, and the person. Wesleyan University Press.

[24] Donald A Schön. 1992. Designing as reflective conversation with the materials of a design situation. Knowledge-based systems 5, 1 (1992), 3-14.

[25] Richard Sennett. 2008. The craftsman. Yale University Press.

[26] Daniel Spelmezan. 2012. An investigation into the use of tactile instructions in snowboarding. In Proceedings of the 14th international conference on Humancomputer interaction with mobile devices and services. 417-426. https://doi.org/ $10.1145 / 2371574.2371639$

[27] Jessi Stark, Fraser Anderson, George Fitzmaurice, and Sowmya Somanath. 2020 MakeAware: Designing to Support Situation Awareness in Makerspaces. In Proceedings of the 2020 ACM Designing Interactive Systems Conference. 1005-1016. https://doi.org/10.1145/3357236.3395472

[28] Brendan Strahm, Colin M Gray, and Mihaela Vorvoreanu. 2018. Generating mobile application onboarding insights through minimalist instruction. In Proceedings of the 2018 Designing Interactive Systems Conference. 361-372. https://doi.org/10. $1145 / 3196709.3196727$

[29] C. L. Teo, E. Burdet, and H. P. Lim. 2002. A robotic teacher of Chinese handwriting In Proceedings 10th Symposium on Haptic Interfaces for Virtual Environment and Teleoperator Systems. HAPTICS 2002. 335-341. https://doi.org/10.1109/HAPTIC 2002.998977

[30] Balasaravanan Thoravi Kumaravel, Fraser Anderson, George Fitzmaurice, Bjoern Hartmann, and Tovi Grossman. 2019. Loki: Facilitating remote instruction of physical tasks using bi-directional mixed-reality telepresence. In Proceedings of the 32nd Annual ACM Symposium on User Interface Software and Technology. 161-174. https://doi.org/10.1145/3332165.3347872

[31] Cesar Torres, Sarah Sterman, Molly Nicholas, Richard Lin, Eric Pai, and Eric Paulos. 2018. Guardians of Practice: A Contextual Inquiry of Failure-Mitigation Strategies within Creative Practices. In Proceedings of the 2018 Designing Interactive Systems Conference. 1259-1267. https://doi.org/10.1145/3196709.3196795

[32] Jeremy Warner, Ben Lafreniere, George Fitzmaurice, and Tovi Grossman. 2018. Electrotutor: Test-driven physical computing tutorials. In Proceedings of the 31st Annual ACM Symposium on User Interface Software and Technology. 435-446. https://doi.org/10.1145/3242587.3242591

[33] Marguerite Wildenhain. 1959. Pottery: Form and expression. American Craftsmen's Council.

[34] Masahiro Yamaguchi, Shohei Mori, Peter Mohr, Markus Tatzgern, Ana Stanescu, Hideo Saito, and Denis Kalkofen. 2020. Video-Annotated Augmented Reality Assembly Tutorials. In Proceedings of the 33rd Annual ACM Symposium on User Interface Software and Technology. 1010-1022. https://doi.org/10.1145/3379337. 3415819

[35] Vibol Yem, Hideaki Kuzuoka, Naomi Yamashita, Ryota Shibusawa, Hiroaki Yano, and Jun Yamashita. 2012. Assisting hand skill transfer of tracheal intubation using outer-covering haptic display. In Proceedings of the SIGCHI Conference on Human Factors in Computing Systems. 3177-3180. https://doi.org/10.1145/ 2207676.2208735 